\title{
Effect of nitric oxide donor and gamma irradiation on modifications of ERK and JNK in murine peritoneal macrophages
}

\author{
Himanshi Narang • Fatema A. Dhariwala • \\ Malini Krishna
}

Received: 27 September 2007 / Accepted: 29 April 2008 / Published online: 4 June 2008

(C) The Author(s) 2008

\begin{abstract}
Mitogen activated protein kinases (MAPKs) play an important role in activation, differentiation and proliferation of macrophages. Macrophages, upon activation, produce large amounts of nitric oxide that inhibit the growth of variety of microorganisms and tumor cells. This nitric oxide which is known to interfere with tyrosine phosphorylation may result in changes in the pattern of activation of MAPKs. In a previous study we have found that tyrosine phosphorylation of MAPKs was completely abolished in the presence of nitric oxide donor and radiation but this did not affect the function of macrophages. In this study the other post translational modifications namely nitration and ubiquitination of JNK and ERK have been looked at. Both ERK and JNK were found to be nitrated. However, there was no increase in ubiquitination of ERK and JNK, indicating that ubiquitination, in this case was not a natural consequence of nitration and may serve in signaling. Additionally, when the nitration was extensive, phosphorylation was also inhibited. The activation of substrates of ERK and JNK were looked at to determine the consequences of such modifications. Inhibition of phosphorylation and extensive nitration of JNK did not prevent activation of its substrate, c-jun. This study indicates that ERK and JNK may be under regulation by different type of modifications in macrophages.
\end{abstract}

Keywords Sodium Nitroprusside (SNP) · Radiation, Mitogen Activated Protein Kinase (MAPK) .

Macrophages and post translational modifications

H. Narang $\cdot$ F. A. Dhariwala $\cdot$ M. Krishna $(\triangle)$

Radiation Biology and Health Sciences Division,

Bhabha Atomic Research Centre,

Mumbai 400085, India

e-mail: malini@barc.gov.in

\section{Introduction}

Signal transduction by mitogen activated protein kinases (MAPKs) is known to involve sequential phosphorylation of tyrosines or serines of the proteins (Martin-Blanco 2000). The last effector in the cascade is a transcription factor, which ultimately binds to DNA and leads to the expression of target genes. The MAP kinase family is composed of the ERK 1/2, p38 and SAPK/JNK pathways and is important for a wide spectrum of cell functions. Although distinct in their activation, there is considerable co-operation between these kinases and many substrates are shared between the pathways (Cobb 1999).

In macrophages, MAPK pathways are activated upon binding of growth factors and inflammatory cytokines to specific receptors and are essential in mediating important cell functions such as proliferation, differentiation (Hu et al. 2000; Das et al. 2000; Valledor et al. 2000), apoptosis (Mohr et al. 1998), cytokine biosynthesis (Kurosawa et al. 2000; Geppert et al. 1994; Kovalovsky et al. 2000), expression of cyclooxygenase-2 and iNOS (Chan et al. 1999; Chen et al. 1999) and phagocytosis (Kugler et al. 1997).

When radiotherapy is delivered to malignant cells, macrophages being radio resistant survive, get activated by radiation and regulate inflammatory and immunological responses. Activated macrophages also produce large amounts of nitric oxide that inhibits the growth of wide variety of tumor cells and microorganisms (MacMicking et al. 1997), and has also been shown to induce apoptosis in macrophages themselves (Albina et al. 1993; Messmer et al. 1995). The activation of MAPKs has been reported to be involved in the apoptotic response to nitric oxide (NO) in macrophage-derived cell lines and MEK inhibitor, inhibitor of upstream activator of ERK, has been shown to protect against cell death after macrophage activation (Mohr et al. 
1998). However many reports have shown the protective effect of NO on macrophages (Yoshioka et al. 2006a, b) and other cells (Davis et al. 2000; Li et al. 1997; Zech et al. 2003). These dual effects of NO on macrophages have been imparted to different concentrations of NO. Low concentrations of NO have been shown to be protective while high concentrations have been shown to be cytotoxic. These varied effects of $\mathrm{NO}$ are mediated via activation or inhibition of signaling components involved, MAPKs being one of them.

With a better understanding of cellular stress response, it has become evident that the catalytic modules consisting of kinases that mediate the activation of downstream effector components are subject to multiple layers of regulation. Many post translation modifications like nitrosylation, nitration and even ubiquitination of signaling proteins seem to play important role in transducing the signal (Di stasi et al. 1999; Lane et al. 2001; Schnell and Hicke 2003; Ischiropoulos 2003). These modifications seem to act by altering the protein stability, localization or protein-protein association.

Nitric oxide is known to interfere with tyrosine phosphorylation and can either diminish the efficacy of a protein as a substrate for tyrosine kinases (Gow et al. 1996; Kong et al. 1996) or lead to their activation by increased phosphorylation directly (Monteiro et al. 2000; Oliveira et al. 2003; Bernabe et al. 2001) or by inactivation of phosphatases (Takakura et al. 1997). Other modifications like S-nitrosylation (Deora et al. 2000; Mondoro et al. 1997) and nitration (Balafanova et al. 2002; Cassina et al. 2000; Vadseth et al. 2004) may also be involved. Nitrated proteins have also been shown to be preferentially degraded via ubiquitin mediated pathways (Souza et al. 2003), hence determining the stability of protein in a cell.

Most of the studies regarding tyrosine nitration and other biological effects are derived from results either by treating macrophages with alkaline solutions of chemically synthesized peroxynitrite, $\mathrm{NO}$ donor or by activating macrophages with LPS/IFN- $\gamma$ which themselves induce many signaling pathways. Simultaneous treatment with NO donor and radiation would be a better approximation in terms of generation of $\mathrm{NO}$ and reactive oxygen species together in macrophages. In this study we have used a small exposure to $\mathrm{NO}$ donor and 2 Gy of gamma radiation dose to simulate the early activation stage of simultaneous NO and ROS production in macrophages while avoiding LPS/IFN $\gamma$ like inducers.

In our earlier study we had observed that when the cells were exposed to both radiation and a nitric oxide donor, phosphorylation of MAPKs was inhibited. The interesting aspect of this study was the fact that the macrophages were functionally unaffected in spite of the inhibition of MAPK phosphorylation (Narang and Krishna 2008). Since the phosphatases were not significantly activated and the total amount of the kinases had not changed, it was of interest to look at the other post translational modification of these kinases. In this study the extent of these modifications in immunoprecipitated kinases (phosphorylation, nitration and ubiquitination) and the subsequent activation of the transcription factors has been looked at in peritoneal macrophages.

\section{Material and methods}

Animals

Male Swiss mice (4-6 weeks) were maintained on a standard laboratory diet with water ad libitum. Animals were reared in polypropylene cages in air-conditioned $(24 \pm$ $2^{\circ} \mathrm{C}$ ) rooms with a 12 -hourly dark and light schedule. Mice used in the present study were a part of conventional inbred colony of Swiss mice maintained at the animal house facility of Bhabha Atomic Research Centre.

All experiments were conducted with strict adherence to the ethical guidelines laid down by the Committee for the Purpose of Control and Supervision of Experiments on Animals (CPCSEA) constituted by the Animal Welfare Division of Government of India on use of animals in scientific research.

\section{Isolation of PEC}

Resident peritoneal exudates cells (PEC) were used as source of macrophages. PEC were isolated from animals by washing out the peritoneal cavity. In brief, RPMI 1640 (Sigma) was injected into peritoneal cavity of mice. After gentle massage of the abdomen, the media was withdrawn with the help of a syringe. The cells were then pelleted by centrifugation at $2500 \mathrm{rpm}$ for $6 \mathrm{~min}$, resuspended in RPMI 1640 medium at concentration of $5 \times 10^{6}$ cells $/ \mathrm{ml}$.

\section{Treatment of PEC}

The cells were divided into four treatment groups which were treated as follows: (a) Controls were untreated. (b) Cells were irradiated (2 Gy, dose rate $0.1 \mathrm{~Gy} / \mathrm{s}$, gamma cell 220 , AECL, Canada) and lysed after 5 or $30 \mathrm{~min}$. (c) One group of cells was treated with SNP $(1 \mathrm{mM})$, one set was lysed after $60 \mathrm{~min}$; two sets were irradiated after $30 \mathrm{~min}$ and lysed after 5 or 30 min respectively. Lysates were either directly processed for SDS-PAGE or were immunoprecipitated.

Immunoprecipitation

Treated or untreated cells $\left(5 \times 10^{6}\right)$ were lysed in cell lysis buffer by using anti rabbit anti-ERK (Sigma) or anti rabbit 
anti-JNK (Sigma) using an immunoprecipitation kit (Sigma). Briefly lysates with protease inhibitors were centrifuged and the supernatants were incubated with anti-ERK or anti-JNK in immunoprecipitation (IP) buffer overnight at $4^{\circ} \mathrm{C}$ with gentle mixing. Protein A Sepharose beads were added and tubes were again incubated for 3 to $4 \mathrm{~h}$ at $4^{\circ} \mathrm{C}$ with gentle mixing. Different concentrations of IP buffer were used for washing the beads to remove unprecipitated protein. Samples were boiled for $10 \mathrm{~min}$ in SDS loading buffer to isolate the immunoprecipitated complex, eluent was collected and directly loaded onto gel for immunoblotting.

\section{SDS-PAGE and Western blot analysis}

For SDS-PAGE, $1 \times 10^{6}$ cells were lysed by boiling in SDS cell lysis buffer [ $10 \%$ glycerol, $2 \%$ SDS, $100 \mathrm{mM}$ DTT, $0.1 \%$ bromophenol blue and $50 \mathrm{mM}$ Tris- $\mathrm{HCl}(\mathrm{pH} \mathrm{6.8)]} \mathrm{for}$ $10 \mathrm{~min}$. Lysates or immunoprecipitates obtained from these lysates as above were then loaded on to $8 \%$ SDS polyacrylamide gel electrophoresis followed by electroblotting onto nitrocellulose membrane (Amersham). After blocking of non-specific binding with 5\% BSA (Sigma), the membranes were probed with the following antibodies. ERK immunoblot was probed with anti mouse anti ERK, anti mouse anti-phosphoERK (Sigma), anti mouse anti-nitrotyrosine, anti mouse anti-ubiquitin (Chemicon). JNK immunoblot was probed with anti mouse anti JNK, anti mouse anti-phosphoJNK (Sigma), anti mouse anti-nitrotyrosine and anti mouse anti-ubiquitin. The immunoblot of cell lysates were probed with anti rabbit phospho c-jun (cell signaling) and anti phospho Elk (cell signaling). This was followed by incubation with horseradish peroxidase (HRP) conjugated secondary anti mouse antibody (Roche Molecular Biochemicals, Germany). The secondary antibody was detected using BM Chemiluminiscence Western Blotting Kit (Roche Molecular Bio Chemicals, Germany). The band intensity was quantified by software Gelquant (version 2.7.0 DNR Bioimaging Systems Ltd.).

\section{Splenic lymphocytes treatment}

Splenic lymphocytes were isolated by making a cell suspension by crushing the spleen on a sieve. The cell suspension was taken as splenic lymphocytes after RBC lysis. Splenic lymphocytes were treated with SNP $(400 \mu \mathrm{M})$ and or irradiation (2 Gy) for time periods as given in figures. After treatment the cells were lysed, processed for immunoblotting and probed with anti-ERK, anti-phosphoERK or anti-phsphoJNK.

All the experiments were carried out three times independently.

\section{Results}

Kinetics of IR and/or SNP induced MAPK activation, nitration and ubiquitination

Figure 1 shows the blots and histograms of tyrosine phosphorylation, nitration and ubiquitination of ERK after irradiation and SNP treatment. Tyrosine phosphorylation of ERK was induced as early as $5 \mathrm{~min}$ (Fig. 1, lane 2). This increase was transient since at $30 \mathrm{~min}$ there was a decline (lane 3). Following SNP treatment alone ERK was partially activated. However extensive irradiation induced phosphorylation observed at 5 min was inhibited with SNP treatment (compare lanes 2 and 5, Fig. 1). At 30 min the phosphorylation recovered but was not as extensive as with radiation alone (lane 6). Tyrosine nitration of ERK was almost absent

\begin{tabular}{|c|c|c|c|c|c|c|}
\hline LANE & 1 & 2 & 3 & 4 & 5 & 6 \\
\hline Phospho ER & - & $=$ & $=$ & & $=$ & - \\
\hline Nitrated ER & & & & & & \\
\hline $\begin{array}{l}\text { Ubiquitinatec } \\
\text { ERK }\end{array}$ & $10=$ & $=0$ & $=$ & 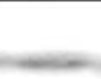 & - & 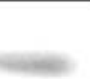 \\
\hline Total & 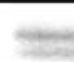 & ments & $m$ & sovit: & 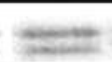 & $=$ \\
\hline $\begin{array}{l}\text { SNP I mM } \\
\text { IR (2Gy) } \\
\text { Time after IR }\end{array}$ & $\begin{array}{l}- \\
-\end{array}$ & $\begin{array}{l}- \\
+ \\
5\end{array}$ & $\begin{array}{l}- \\
+ \\
30\end{array}$ & $\begin{array}{l}+ \\
- \\
-\end{array}$ & $\begin{array}{l}+ \\
+ \\
5\end{array}$ & $\begin{array}{l}+ \\
+ \\
30\end{array}$ \\
\hline
\end{tabular}

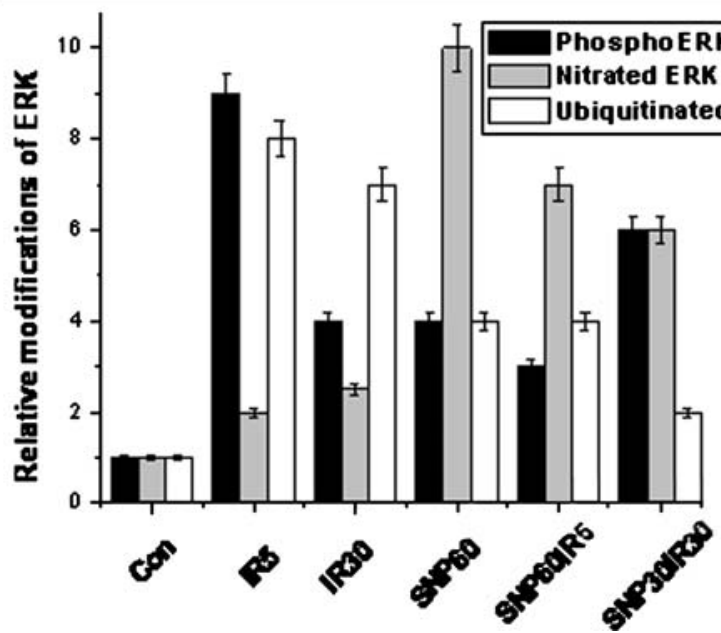

Fig. 1 Effect of SNP and/or IR treatment on tyrosine phosphorylation, tyrosine nitration and ubiquitination of ERK1/2: PEC were irradiated (2 Gy) and lysed after 5 and $30 \mathrm{~min}$ in the absence or presence of SNP $(1 \mathrm{mM}, 60 \mathrm{~min})$. The total ERK was immunoprecipitated from the lysates with anti rabbit anti ERK followed by immunoblotting. Membranes were then probed by anti mouse anti ERK, anti phospho-ERK, anti-nitrotyrosine or anti-ubiquitin. a One of three resulting hybridization images of each is shown here. b Histogram representing relative intensities of phospho ERK, Nitrated ERK and ubiquitinated ERK. Results are representative of three independent experiments 
in controls and increased extensively by treatment with SNP (nitrated ERK, lanes 1 and 4). SNP treated cells that were irradiated also showed considerable nitration of ERK (Fig. 1, nitrated ERK, lanes 5 and 6). ERK was found to be ubiquitinated appreciably with IR treatment (lane 2 and 3). SNP treatment also resulted in increased ubiquitination as compared to control. However when SNP treatment was followed by irradiation (lane 5 and 6 ), there was a time dependent decrease in ubiquitination, as compared to IR (lane 2 and 3) or SNP alone (lane 4).

Figure 2 shows the blots and representative histograms of tyrosine phosphorylation, nitration and ubiquitination of

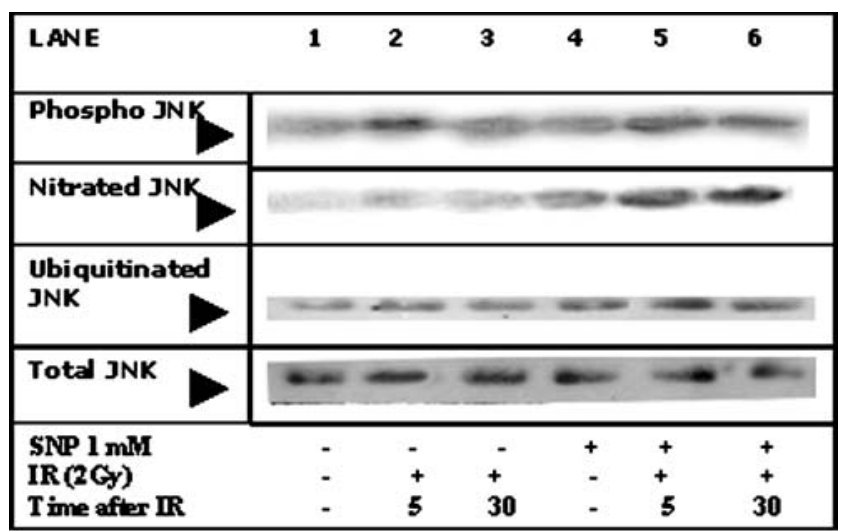

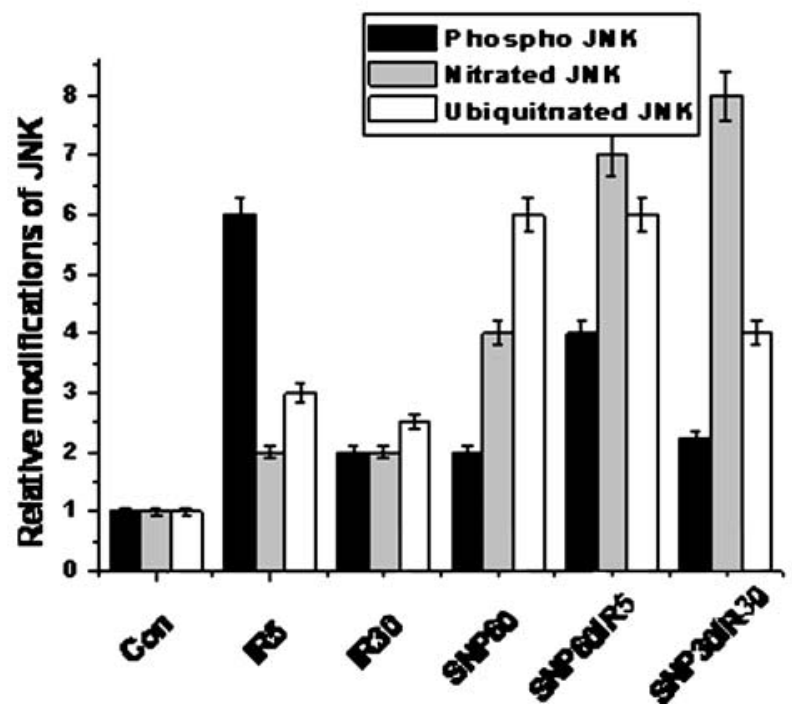

Fig. 2 Effect of SNP and/or IR treatment on tyrosine phosphorylation, tyrosine nitration and ubiquitination of JNK: PEC were irradiated ( 2 Gy) and lysed after 5 and $30 \mathrm{~min}$ in the absence or presence of SNP ( $1 \mathrm{mM}, 60 \mathrm{~min}$ ). The total JNK was immunoprecipitated from the lysates with anti rabbit anti JNK followed by immunoblotting. Membranes were then probed by anti mouse anti JNK, anti phospho-JNK, anti-nitrotyrosine or anti-ubiquitin. a One of three resulting hybridization images of each is shown here. b Histogram representing relative intensities of phospho JNK, Nitrated JNK and ubiquitinated JNK. Results are representative of three independent experiments
JNK after irradiation and SNP treatment. Like ERK, JNK also showed extensive phosphorylation at $5 \mathrm{~min}$ after irradiation and then a decline (lane 2 and 3). SNP treatment inhibited the irradiation induced increase in phosphorylation but extensively increased JNK's nitro tyrosine immunoreactivity which was not transient like its phosphorylation (Fig. 2, lane 5 and 6). Tyrosine nitration of JNK also increased in presence of SNP alone (lane 4). JNK was found to be ubiquitinated appreciably with SNP treatment (lane 4). However when SNP treatment was followed by irradiation, there was a time dependent decrease in ubiquitination, as compared to SNP alone (lane 5 and 6).

The total ERK and JNK as observed in immunoprecipitated lysates was unchanged with SNP treatment alone or followed by irradiation and has been used as a control for immunoprecipitation (Figs. 1 and 2).

\section{Estimation of ERK and JNK activities}

Figure 3 shows the phosphorylation of Elk and c-jun which are substrates for ERK and JNK respectively and hence are the indicators of their activities. A high basal phosphorylation of elk which decreased 5 min after irradiation and then came back to control levels after 30 min was observed (lanes 2 and 4, Fig. 3a). This did not conform to the phosphorylation status of ERK and was in fact inverse of that. Treatment with both SNP and IR also led to a decrease in phosphorylation of elk (lanes 5 and 6, Fig. 3a).

c-jun showed increased phosphorylation after irradiation which was persistent till 30 min unlike the activation of its precursor, JNK (Fig. 3a, lanes 2 and 4; Fig. 3c). The IR induced phosphorylation of c-jun was further enhanced by pretreatment with SNP for 30 min indicating that in spite of inhibition of phosphorylation of its precursor, the transcription factor jun could be activated (lanes 5 and 6 ).

Splenic lymphocytes MAPK phosphorylation after SNP/IR treatment

It was likely that the inhibition of phosphorylation by SNP observed in this study could be characteristic of macrophages since they are NO producing cells and are habituated to operating in an NO environment and that other cells may not respond to SNP in the same way. To test this hypothesis the splenic lymphocytes were treated in the same way i.e. treated with SNP prior to irradiation and the phosphorylation of the ERK was looked at in the lysate (Fig. 4). The response of the ERK was similar to the macrophages and was more prominent. ERK showed considerable inhibition of irradiation induced phosphorylation after SNP treatment (compare lanes 3 and 4, 6 and 7, 9 and 10) but unlike in the macrophages, does not recover its phosphorylation even after $180 \mathrm{~min}$. 


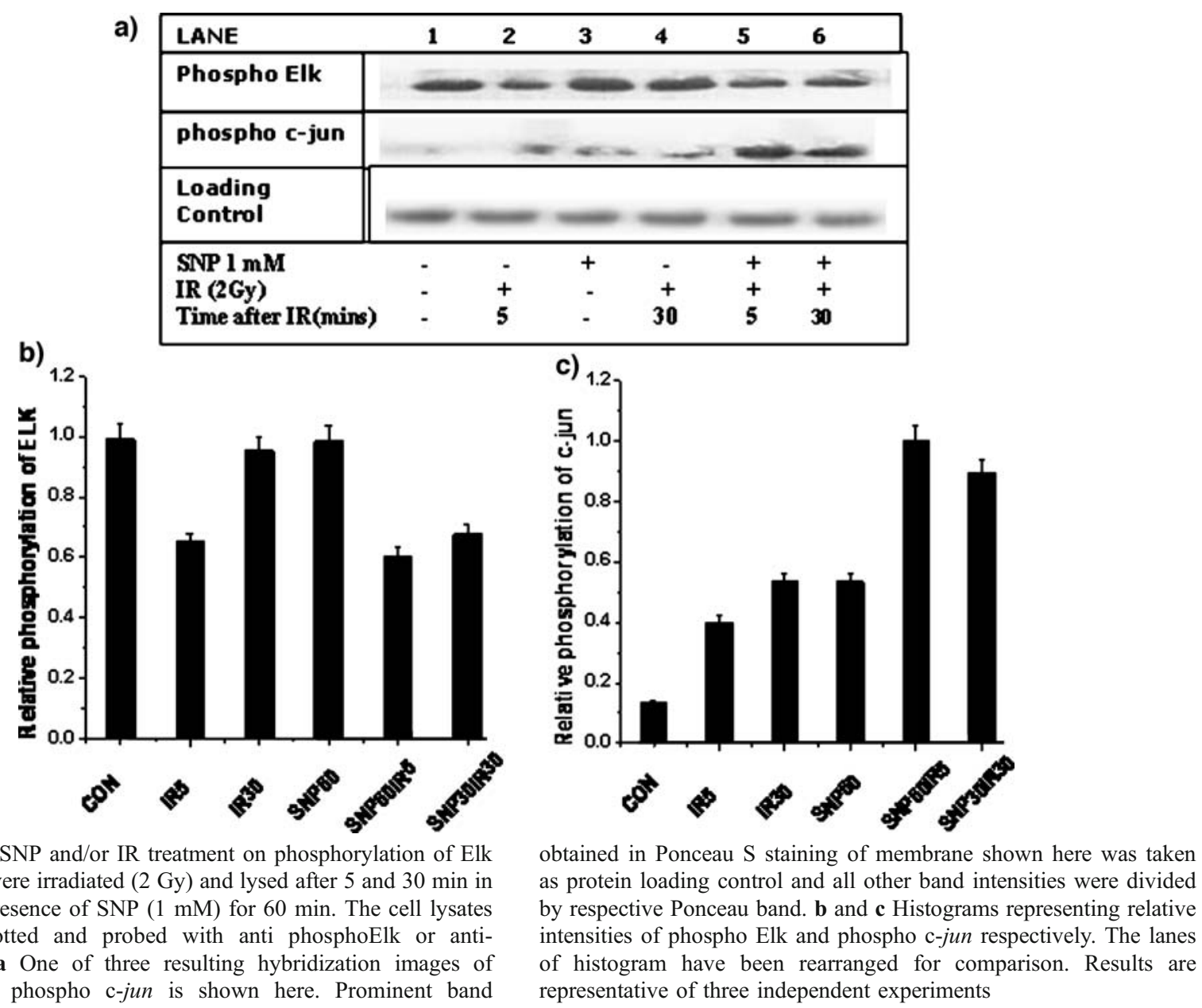

\section{Discussion}

MAPKs have been implicated in proliferation, differentiation (Das et al. 2000) and activation (Carter et al. 1999) of macrophages. MAPK pathways are known to get activated upon irradiation in many cells including macrophages (Dent et al 2003). In our earlier study we had observed that exposure of macrophages to SNP, a NO donor, led to a complete inhibition of the radiation induced phosphorylation of ERK and JNK. Despite the complete inhibition of MAPK phosphorylation; the viability, caspase 3 activity and phagocytic efficiency of macrophages were unaffected indicating that MAPK signaling was still operative (Narang et al. 2008). Since SNP is a nitric oxide donor, which in presence of radiation forms nitrating species, it is quite likely that the MAPKs are nitrated at tyrosines. In this study, the tyrosine nitration and ubiquitination of these MAPKs was looked at. The phosphorylation, nitration and ubiquitination of ERK and JNK were observed at 0,5 and $30 \mathrm{~min}$. Both ERK and JNK were activated with radiation dose of 2 Gy as early as 5 min and then showed a decline. But exposure to an NO donor led to inhibition of radiation induced phosphorylation of ERK and JNK (Figs. 1 and 2,

\begin{tabular}{|c|c|c|c|c|c|c|c|c|c|c|}
\hline LANB & 1 & 2 & 3 & 4 & 5 & 6 & 7 & 8 & 9 & 10 \\
\hline phosphoERK & z & & $=$ & - & - & $\Rightarrow$ & - & & - & \\
\hline Total ERK & 5 & & & & & & & & 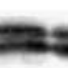 & $=8$ \\
\hline Loading Control & - & $=$ & $=$ & $=$ & $=$ & - & - & 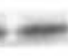 & - & - \\
\hline $1 \mathrm{mM} \mathrm{SNP}$ & - & + & - & + & + & - & + & + & - & + \\
\hline IR & - & - & + & + & - & + & + & - & + & + \\
\hline Time after $\mathbf{I}$ (mins) $_{\text {(in }}$ & - & - & 5 & 5 & - & 30 & 30 & - & 180 & 180 \\
\hline Time in SNP(mins) & - & 60 & - & 60 & 90 & - & 90 & 240 & - & 240 \\
\hline
\end{tabular}

Fig. 4 Effect of SNP and/or irradiation with respect to time of treatment on phosphorylation status of ERK in splenic lymphocytes: $10^{6}$ cells $/ \mathrm{ml}$ were treated with radiation dose of 2 Gy or with SNP $(400 \mu \mathrm{M})$ and then lysed at indicated time periods (5, 30 and $180 \mathrm{~min}$ ); lanes 3, 6 and 9. One set of cells was pretreated with SNP $(0.4 \mathrm{mM})$ for $60 \mathrm{~min}$ before irradiation and then lysed after 5, 30 and $180 \mathrm{~min}$; lanes 4, 7 and 10. SNP treatment alone was given for 60,90 and $240 \mathrm{~min}$; lane 2, 5 and 8 . One of three resulting hybridization images of phospho-ERK and total ERK are shown here. Prominent band obtained in $1 \%$ Ponceau staining of the membrane is given as protein loading control 
phospho ERK and JNK, compare lanes 2, 3 with 5, 6). Treatment with an NO donor led to increase in tyrosine nitration which further increased when NO treated macrophages were irradiated (Figs. 1 and 2, nitrated ERK and JNK, lanes 3-5). This could be because the reactive oxygen species generated following irradiation react with nitric oxide to generate reactive nitrogen species like peroxynitrite (Reiter et al. 2000) which are capable of nitrating the tyrosine residues in proteins. The nitration and phosphorylation did not seem to take place simultaneously in the kinases. At the time points when the nitration is extensive, phosphorylation is partially inhibited (Figs. 1 and 2, lanes 5 and 6).

Ubiquitination was looked for to check if they had been tagged for degradation. The results show that ubiquitination did not go hand in hand with nitration. Ubiquitination was in fact inhibited when macrophages were treated with both SNP and radiation as compared to treatment with SNP alone. However, it's been recognized that ubiquitination not only just tags the proteins for degradation but may play important role as a modification controlling various aspects of signaling like duration, magnitude or localization (Schnell and Hicke 2003; Di Fiore et al. 2003). MAPK pathways also have been shown to be under regulation by ubiquitination where MEKK, ubiquitinates its precursors including c-jun and ERK1c dictating their stability and localization respectively (Laine and Ronai 2005). That ubiquitination is not taking these nitrated proteins (ERK and JNK) for degradation is evident from the fact that following irradiation and SNP their total content did not change. However, since ubiquitination of these proteins is yet operative, it may play a role in regulation of MAPK signaling.

We further looked at the transcription factors acted upon by these kinases to see the translation of the radiation and nitric oxide stress on the effectors of these pathways. Though Elk and c-jun were initially known to be preferred targets of ERK and JNK respectively (Shaulin and Karin 2001; Davis 2000), the emerging studies of crosstalk between these pathways have blurred the specificity of substrate assigned to each. Many reports have shown the phosphorylation of elk by JNK (Zhang et al. 2007; Yang et al. 1998) and vice versa i.e. phosphorylation of c-jun by ERK (Leppa et al. 1998).

In our studies we looked at the phosphorylation of these downstream effectors, elk and c-jun, where c-jun was not phosphorylated to large extent after radiation or nitric oxide alone. But after both radiation and nitric oxide treatment, phosphorylation of c-jun increased significantly while elk showed decrease in phosphorylation. Previous studies have also shown that pro-survival ERK pathway and proapoptotic JNK pathway act in dynamic balance with respect to radiation exposure, with one pathway excluding the activation of other (Carter et al. 1998; Reardon et al. 1999) also sustained activation of JNK is known to block ERK activation by mitogenic factors. In this study we have observed this shifting of phosphorylation from elk to c-jun between the two pathways with respect to dual stress (radiation and nitric oxide). This is logical since the two stresses need to be indicated and translated into effect in the cell via c-jun activation which is a stress responsive factor (Hibi et al. 1993; Derijard et al. 1994). Moreover, these transcription factors are not known to act in an independent manner but in association with other proteins (Karin et al. 1997). The oligomeric complex that forms by association of two or more transcription factors regulates different target genes and executes different biological functions depending upon its constituents.

After both the stresses at $60 \mathrm{~min}$, when phosphorylation of JNK is inhibited and its nitration extensive, the phosphorylation of c-jun was significantly high (Fig. 3a, lanes 5 and 6). This indicates that nitrated JNK may be capable of transmitting a signal as effectively as its phosphorylated counterpart. Although both ERK and JNK were nitrated, only c-jun was phosphorylated, elk was not. This might indicate the specificity of regulation by nitration where it inhibits one kinase while activating the other.

Multiple modifications are a normal mode of signal transduction in physiology. Besides phosphorylation, nitration and nitrosylation of many signaling proteins has been shown to activate them, e.g. src (Macmillan-Crow et al. 2000) and many neurosignaling proteins (Jaffery et al. 2001). Tyrosine nitration has been shown to activate protein kinase $\mathrm{C}$ epsilon (Balafanova et al. 2002), cytochrome c (Cassina et al. 2000) and fibrinogen (Vadseth et al. 2004). Many crucial proteins are also known to undergo nitration with a change in their properties (Yamakura et al. 1998; Hellberg et al. 1998 and Macmillan-Crow et al. 2000) and also their biological half life. Nitrosylation of $\mathrm{bcl}_{2}$ inhibits its ubiquitin mediated proteasomal degradation (Azad et al. 2006) while nitration of FLICE increases its proteasomal degradation (Chanvorachote et al. 2005).

In this study the nitration of JNK may extend its biological half life such that the signal is prolonged or is amplified which is reflected in the extensive phosphorylation of its substrate, cjun. Many studies have established that time-course of activation of ERK and JNK plays important role in deciding the final outcome of the cell e.g. in the macrophages time course of ERK takes it either towards proliferation or differentiation (Valledor et al. 2000). Following radiation damage also it has been shown that transient activation of ERK led to proliferation whereas persistent activation led to cell cycle arrest followed by apoptosis (Deak et al. 1998; Poon et al. 1996). In Jurkat cells persistent but not transient activation of JNK led to apoptosis (Faris et al. 1998). That the life of these signaling proteins has been extended is evident by the fact that their ubiquitination and hence the ensuing degradation are inhibited. 
In conclusion, MAPKs may be under regulation of different post translational modifications during initial stage of nitric oxide release during macrophage activation, three of which have been dealt with in this study. This interplay might affect time course of their activation. However, it is quite likely that other post translational modification, yet unknown, may also participate in signaling.

Open Access This article is distributed under the terms of the Creative Commons Attribution Noncommercial License which permits any noncommercial use, distribution, and reproduction in any medium, provided the original author(s) and source are credited.

\section{References}

Albina JE, Cui S, Mateo RB, Reichner JS (1993) Nitric oxide mediated apoptosis in murine peritoneal macrophages. J Immunol 150:5080-5085

Azad N, Valayathan V, Wang L, Tantishaiyakul V, Stehlik C, Leonard SS, Rojanasakul Y (2006) S-nitrosylation of Bcl-2 inhibits its ubiquitin-proteasomal degradation. A novel antiapoptotic mechanism that suppresses apoptosis. J Biol Chem 281(45):3412434134

Balafanova Z, Bolli R, Zhang J, Zheng Y, Pass JM, Bhatnagar A, Tang XL, Wang O, Cardwell E, Ping P (2002) Nitric oxide (NO) induces nitration of protein kinase $\mathrm{C}$ e (PKC e), facilitating PKC epsilon translocation via enhanced PKCepsilon-RACK2 interactions: a novel mechanism of NO-triggered activation of PKCepsilon. J Biol Chem 277(17):15021-15027

Baldassare JJ, Bi Y, Bellone CJ (1999) The role of p38 mitogenactivated protein kinase in IL-1 beta transcription. J Immunol 162:5367-5373

Bernabe JC, Tejedo JR, Rincon P, Cahuana GM, Ramirez R, Sobrino Fi, Bedoya FJ (2001) Sodium nitroprusside-induced mitochondrial apoptotic events in insulin secreting RINm5F cells are associated with MAP kinases activation. Exp Cell Res 269:222229

Carter AB, Monick MM, Hunninghake GW (1999) Both Erk and p38 kinases are necessary for cytokine gene transcription. Am J Respir Cell Mol Biol 20:751-758

Carter S, Auer KL, Reardon DB, Birrer M, Fisher PB, Valerie K, Schmidt-Ulrich R, Mikkelson R, Dent P (1998) Inhibition of mitogen activated protein (MAP) kinase cascade potentiates cell killing by low dose ionizing radiation in A431 human squamous carcinoma cells. Oncogene 16:2787-2796

Cassina AM, Hodara R, Souza JM, Thomson L, Castro L, Ischiropoulos H, Freeman BA, Radi R (2000) Cytochrome c nitration by peroxynitrite. J Biol Chem 275:21409-21415

Chan ED, Winston BW, Uh ST, Remigio LK, Riches DW (1999) Systemic evaluation of the mitogen activated protein kinase in the induction of iNOS by tumor necrosis actor-alpha and IFNgamma. Chest 116:91S-92S

Chanvorachote P, Nimmannit U, Wang L, Stehilk C, Lu B, Azad N, Rojanasakul Y (2005) Nitric oxide negatively regulates Fas CD95-induced apoptosis through inhibition of ubiquitin-proteasome-mediated degradation of FLICE inhibitory protein. J Biol Chem 280(51):42044-42050

Chen C, Chen YH, Lin WW (1999) Involvement of p38 mitogen activated protein kinase in lipopolysaccharide-induced iNOS and COX-2 expression in J774 macrophages. Immunology 97:124-129
Cobb MH (1999) MAP kinase pathways. Prog Biophys Mol Biol 71:479-500

Das D, Pintucci G, Stern A (2000) MAPK-dependent expression of p21 (WAF) and p27 (kip1) in PMA induced differentiation of HL60 cells. FEBS Lett 472:50-52

Davis DW, Weidner DA, Holian A, McConkey DJ (2000) Nitric oxide dependent activation of p53 suppresses bleomycin induced apoptosis in lung. J Exp Med 192:857-869

Davis RJ (2000) Signal transduction by the JNK group of MAP kinases. Cell 103:239-252

Deak M, Clifton AD, Lucoco LM, Alessi DR (1998) Mitogen- and stress-activated protein kinase-1 (MSK1) is directly activated by MAPK and SAPK2/p38, and may mediate activation of CREB. EMBO J 15:4426-4441

Dent P, Yacoub A, Fisher PB, Hagan MP, Grant S (2003) MAPK in radiation responses. Oncogene $22: 1-12$

Deora AA, Hajjar DP, Lander HM (2000) Recruitment and activation of Raf-1 kinase by nitric oxide activated Ras. Biochemistry 39:9901-9908

Derijard B, Hibi M, Wu IH, Barrett T, Su B, Deng T, Karin M, Davis RJ (1994) JNK1: a protein kinase stimulated by UV light and HaRas that binds and phosphorylates the c-jun activation domain. Cell 76:1025-1037

Di Fiore PP, Polo S, Hofmann K (2003) When ubiquitin meets ubiquitin receptors: a signaling connection. Nat Rev Mol Cell Bio 4:491-497

Di stasi AMM, Mallozzi C, Macchia G, Petrucci TC, Minetti M (1999) Peroxynitrite induces tyrosine nitration and modulates tyrosine phosphorylation of synaptic proteins. J Neurochem 73:727-735

Faris M, Kokot N, Latinis K, Kasibhalta S, Green DR, Koretzky GA, Nel A (1998) The c-Jun N-terminal kinase cascade plays a role in stress-induced apoptosis in Jurkat cells by up-regulating Fas ligand expression. J Immunonol 160:134-144

Geppert TD, Whitehurst CE, Thompson P, Beutler B (1994) Lipopolysaccharide signals activation of tumor necrosis factor biosynthesis through Ras/raf/MEK/MAP pathway. Mol Med 1:93-103

Gow AJ, Duran D, Malcom S, Ischripoulos H (1996) Effects of peroxynitrite-induced protein modifications on tyrosine phosphorylation and degradation. FEBS Lett 385:63-66

Hellberg CB, Bogs SE, Lapetina EG (1998) Phosphatidylinositol 3kinase is a target for protein tyrosine nitration. Biochem Biophys Res Commun 252:313-317

Hibi M, Lin A, Smeal T, Minden A, Karin M (1993) Identification of an oncoprotein and UV responsive protein kinase that binds and potentiates the c-jun activation domain. Genes Dev 7:21352148

Hu X, Moscinski LC, Valkov NI, Fisher AB, Hill BJ, Zuckerman KS (2000) Prolonged activation of the mitogen activated protein kinase pathway is required for macrophage like differentiation of human myeloid leukemic cell line. Cell Growth Differ 11:191200

Ischiropoulos H (2003) Biological selectivity and functional aspects of protein tyrosine nitration. Biochem Biophy Res Commun 305:776-783

Jaffery SR, Erdjument-Bromage H, Ferris CD, Tempst P, Snyder SH (2001) Protein S-nitrosylation: a physiological signal for neuronal nitric oxide. Nat Cell Biol. 3:193-197

Karin M, Liu Z, Zandi E (1997) AP-1 function and regulation. Curr Opin Cell Biol 9(2):240-246

Kong SK, Yim M, Stadman ER, Chock PB (1996) Peroxynitrite disables the tyrosine phosphorylation regulatory mechanism: lymphocyte specific tyrosine kinase fails to phosphorylate nitrated cdc2 (6-20) peptide. Proc Natl Acad Sci U S A 93:3377-3382 
Kovalovsky D, Refojo D, Holsboer F, Arzt E (2000) Molecular mechanisms and Th1/Th2 pathways in corticosteroid regulation of cytokine production. J Neuroimmunol 109:23-29

Kugler S, Schuller S, Goebel W (1997) Involvement of MAP kinases and phosphatases in uptake and intracellular replication of listeria monocytogens in J774 macrophage cells. FEMS Microbiol Lett 157:131-136

Kurosawa M, Numazawa S, Tani Y, Yoshida T (2000) ERK signaling mediates the induction of inflammatory cytokines by bufalin in human monocytic cells. Am J Physiol Cell Physiol 278:C500-C508

Laine A, Ronai Z (2005) Ubiquitin chains in the ladder of MAPK signaling. Sci STKE 281:re5

Lane P, Hao G, Gross SS (2001) S-nitrosylation is emerging as a specific and fundamental posttranslational protein modification: head to head comparison with O-phosphorylation. Sci STKE 86:re1

Leppa S, Saffrich R, Ansrge W, Bohmann D (1998) Differential regulation of c-Jun by ERK and JNK during PC12 cell differentiation. EMBO J 17:4404-4413

Li J, Billar TR, Talanian RV, Kim TR (1997) Nitric oxide reversibly inhibits seven members of caspase family via S-nitrosylation. Biochem Biophys Res Commun 240:419-424

MacMicking J, Xie QW, Nathan C (1997) Nitric oxide macrophage function. Annual Rev Immunol 15:323-350

Macmillan-Crow LA, Greendorfer JS, Vickers SM, Thompson JA (2000) Tyrosine nitration of c-src tyrosine kinase in human pancreatic ductal adenocarcinoma. Arch Biochem Biophy 377(2):350-356

Mallozzi C, Di stasi AMM, Minetti M (1999) Activation of src tyrosine kinases by peroxynitrite. FEBS lett 456:201-206

Martin-Blanco E (2000) p38 MAPK signaling cascades: ancient roles and new functions. Bioessays 22:637-645

Messmer UK, Lapetina EG, Brune B (1995) Nitric oxide induced apoptosis in RAW264.7 macrophages is antagonized by PKC and PKA activating compounds. Mol Pharmacol 47:757-765

Mohr S, McCormick TS, Lapetina EG (1998) Macrophages resistant to endogenously generated nitric-oxide apoptosis are hypersensitive to exogenously added nitric oxide donors: Dichotomous apoptotic response independent of caspase 3 and reversal by mitogen activated protein kinase inhibitor PD098059. Proc Natl Acad Sci U S A 95:5045-5050

Mondoro TH, Shafer BC, Vostal JG (1997) Peroxynitrite-induced tyrosine nitration and phosporylation in human platelets. Free Radical Biol Med 22:1055-1063

Monteiro HP, Gruia-Gray J, Peranovich TMS, Barbosa deOliviera LC, Stern A (2000) Nitric oxide stimulates tyrosine phosphorylation of focal adhesion kinase, Src Kinase and mitogen activated protein kinases in murine fibroblasts. Free Rad Biol Med 28:174-182

Narang H, Krishna M (2008) Effect of nitric oxide donor and gamma irradiation on MAPK signaling in murine peritoneal macrophages. J Cell Biochem 103(2):576-587

Oliveira CJR, Schindler F, Ventura AM, Morais MS, Arai RJ, Debbas V, Stern A, Monteiro HP (2003) Nitric oxide and cGMP activate the Ras-MAPK pathway stimulating protein tyrosine phosphorylation in rabbit aortic endothelial cells. Free Rad Biol Med 35:381-396

Poon RYC, Jiang W, Toyoshima H, Hunter T (1996) Cyclindependent kinases are inactivated by a combination of $\mathrm{p} 21$ and Thr-14/Tyr-15 phosphorylation after UV-induced DNA damage. J Biol Chem 271:13283-13291
Reardon DB, Contessa J, Mikkelson R, Valerie K, Amir C, Dent P, Schmidt-Ulrich RK (1999) Dominant negative EGFR-CD533 and inhibition of MAPK modify JNK1 activation and enhance radiation toxicity of human mammary carcinoma cells. Oncogene 18:4756-4766

Reiter CD, Teng RJ, Beckman JS (2000) Superoxide reacts with nitric oxide to nitrate tyrosine at physiological $\mathrm{pH}$ via peroxynitrite. J Biol Chem 275:32460-32466

Schnell JD, Hicke L (2003) Non-traditional functions of ubiquitin and ubiquitin-binding proteins. J Biol Chem 278(38):3585735860

Shaulin E, Karin M (2001) AP-1 in cell proliferation and survival. Oncogene 20:2390-2400

Souza JM, Choi I, Chen Q, Weisse M, Daikhin E, Yudkoff M, Obin M, Ara J, Horwitz J, Ischripoulos H (2003) Proteolytic degradation of nitrated proteins. Arch Biochem Biophys 380:360-366

Takakura K, Beckman JS, Mac-millan-Crow LA, Crow JP (1997) Rapid and irreversible inactivation of phosphatases PTP1B, CD45 and LAR by peroxynitrite. Arch Biochem Biophys 369:197-207

Vadseth C, Souza JM, Thomson L, Seagraves A, Nagaswami C, Scheiner T, Torbet J, Vilaire G, Bennett JS, Murciano JC, Muzykantov V, Penn MS, Hazen SL, Weisel JW, Ischiropoulos H (2004) Pro-thrombotic state induced by post-translational modification of fibrinogen by reactive nitrogen species. J Biol Chem 279(10):8820-8826

Valledor AF, Comalada M, Xaus J, Caleda A (2000) The differential time course of extracellular-regulated kinase activity correlates with the macrophage response toward proliferation or activation. J Biol Chem 275:7403-7409

Vinciguerra M, Vivacqua A, Fasanella G, Gallo A, Cuozzo, Morano A, Maggiolini M, Musti AM (2004) Differential phosphorylation of c-jun and JUND in response to the epidermal growth factor is determined by the structure of MAPK targeting sequences. J Biol Chem 279(10):9634-9641

Vrana JA, Grant S, Dent P (1999) Inhibition of the MAPK pathway abrogates BCL2-mediated survival of leukemia cells after exposure to low-dose ionizing radiation. Radiat Res 151:559-569

Yamakura F, Taka H, Fujimura, Murayama T (1998) Inactivation of human Mn superoxide dismutase by peroxynitrite is caused by exclusive nitration of tyrosine 34 to 3-nitrotyrosine. J Biol Chem 273:14085-14089

Yang SH, Whitemarsh AJ, Davis RJ, Sharrocks AD (1998) Differential targeting of MAP kinases to the ETS-domain transcription factor Elk-1. EMBO J 17(6):1740-1749

Yoshioka Y, Kitao T, Kishino T, Yamamura A, Maeda S (2006a) Nitric oxide protects macrophages from hydrogen peroxide induced apoptosis by inducing the formation of catalase. $\mathrm{J}$ Immunol 176:4675-4681

Yoshioka Y, Yamamuro A, Maeda S (2006b) Nitric oxide/cGMP signaling pathway protects RAW264 cells against nitric oxide induced apoptosis by inhibiting the activation of p38 mitogen activated protein kinase. J Pharmacol Sci 101:126-134

Zhang L, Yang SH, Sharrocks AD (2007) rev7/MAD2B links c-jun $\mathrm{N}$-terminal protein kinase pathway signaling to activation of the transcription factor Elk-1. Mol Cell Biol 27(8):2861-2869

Zech B, Kohl R, Von knethen A, Brune B (2003) Nitric oxide donors inhibit formation of the Apaf-1/caspase-9 apoptosome and activation of caspases. Biochem J 371:1055-1064 